\title{
Interférences
}

Ars scribendi

$8 \mid 2015$

L'exil au miroir de la Correspondance de Cicéron

\section{Absence et présence dans les lettres d'exil de Cicéron}

Jean-Pierre De Giorgio

\section{(2) OpenEdition}

Journals

Édition électronique

URL : http://journals.openedition.org/interferences/5468

DOI : 10.4000/interferences.5468

ISSN : $1777-5485$

Éditeur

HiSoMA - Histoire et sources des Mondes antiques

Référence électronique

Jean-Pierre De Giorgio, «Absence et présence dans les lettres d'exil de Cicéron », Interférences [En ligne], 8 | 2015, mis en ligne le 03 novembre 2014, consulté le 15 septembre 2020. URL : http:// journals.openedition.org/interferences/5468; DOI : https://doi.org/10.4000/interferences.5468

Ce document a été généré automatiquement le 15 septembre 2020

Tous droits réservés 


\title{
Absence et présence dans les lettres d'exil de Cicéron
}

\author{
Jean-Pierre De Giorgio
}

1 Le titre de cette étude fait écho à celui d'un article de Patrizia Violi ${ }^{1}$ datant de 1988, dans un ouvrage collectif consacré aux approches sémiotiques de l'épistolaire, entendu comme genre de discours davantage que comme genre littéraire: «Présence et absence. Stratégies d'énonciation de la lettre». L'auteur cherchait à déterminer la spécificité énonciative de toute communication épistolaire, l'inscription du «narrateur » étant plus nécessaire que dans n'importe quel autre type de discours, du fait de l'écart spatio-temporel. La lettre apparaissait alors comme «un échange communicatif caractérisé par l'absence de l'interlocuteur. C'est de ce trait propre au genre épistolaire que dérivent certains éléments structuraux caractérisant les formes de l'énonciation et donc les modalités qui sous-tendent la manifestation de la subjectivité dans le texte, modalités caractérisées [...] par une polarité entre absence et présence ${ }^{2}$.» L'intérêt de la démarche tenait d'abord au fait que la tension entre absence et présence était interprétée du point de vue du système énonciatif ${ }^{3}$, dans la perspective d'une linguistique de l'énonciation ouverte, en France, avec Charles Bally ${ }^{4}$ puis, surtout, avec Émile Benvéniste ${ }^{5}$ qui, cependant, définissait l'énonciation à partir de l'«ici et maintenant" de l'énonciateur et de son interlocuteur. L'écart spatiotemporel constitutif de l'écriture d'une lettre en détermine profondément la structure énonciative, mais aussi thématique. L'on s'écrit parce qu'on est éloigné et, parfois, on écrit le fait d'être éloigné, comme dans les lettres de voyage, d'exil, les lettres amicales ${ }^{6}$. Dans le sillage de ces approches, développées ensuite par l'Analyse du discours ${ }^{7}$, les conséquences formelles, thématiques et énonciatives de cette parole in absentia dans l'épistolographie antique ont à présent été bien étudiées ${ }^{8}$.

De fait, cette caractérisation de la lettre comme énoncé entre présence et absence hérite d'une tradition plus ancienne que la linguistique énonciative. L'absence était déjà au cœur de la définition de la lettre par Cicéron. Celle-ci, dans les Philippiques, est interprétée comme une "conversation des amis absents» (ou séparés) : amicorum colloquia absentium (Phil. 4, 7). La naissance même de la lettre, écrit par ailleurs le même 
Cicéron au jeune Curion, serait liée à la nécessité d'informer les absents ${ }^{9}$ : le couple absence/présence entre donc dans la définition de l'échange comme réalisation d'un officium, d'un devoir lié à l'amicitia ${ }^{10}$. Il est aussi un lieu commun de la conversation à distance entre amis, à Rome, largement exploité par Cicéron.

Or, si cette approche permet de caractériser toute pratique de l'écriture épistolaire, elle prend un relief particulier dans le cadre des lettres d'exil de Cicéron. Celui-ci, prenant l'initiative de s'éloigner de Rome, d'abord, pense-t-il, pour quelques jours ${ }^{11}$, se sentant visé par une rogatio ${ }^{12}$ de Clodius (de capite ciuis Romani, affichée en février), puis empêché de revenir par une seconde loi (souvent appelée de exsilio Ciceronis) prescrivant explicitement, par contumace, l'interdictio aqua et igni, est contraint de s'établir à plus de 500 milles de Rome durant près de seize mois. S'il part de lui-même, sans doute avant même que la première loi ne soit effectivement votée, il ne peut revenir sans danger et sans déshonneur. La tension entre absence et présence prend alors une dimension particulière. La lettre n'est plus l'expression d'un simple écart spatiotemporel entre deux locuteurs communiquant par écrit ou un topos de la conversation entre amis séparés. Elle est l'expression d'une contrainte spécifique s'appliquant à l'un des deux correspondants seulement. La correspondance de l'exil ne s'interprète alors pas tant comme une conversation des absents que comme le discours - voire, parfois, le monologue - d'un absent. L'absence se définit en effet dans ces lettres d'abord par l'impossibilité d'être à Rome, seul espace capable de conférer une visibilité et une identité civique: les correspondants peuvent espérer se revoir, mais, comme le souligne constamment Cicéron, pas à Rome. L'épistolaire, dans les lettres d'exil, ne traduit pas seulement une tension entre absence et présence entre les correspondants, mais aussi entre l'épistolier et Rome, entre l'épistolier et lui-même. C'est dans cette perspective que les lettres d'exil posent le problème de l'identité et de la figuration de soi de celui qui, loin de Rome et ayant perdu la face, refuse de se résoudre à se consoler ou à être consolé en « intensifiant ", pour reprendre une expression foucaldienne, «la présence à soi-même " ${ }^{13}$, en nourrissant une indifférence toute philosophique aux revers de la fortune et en relativisant le jeu du pouvoir, ce qu'il prétendra pourtant savoir faire dans d'autres périodes de son existence ${ }^{14}$. Pour Cicéron, l'identité demeure encore largement indissociable de sa présence symbolique et effective à Rome.

\section{L'épistolaire et la théorie de la conversation des absents}

4 Le couple absence/présence apparaît très souvent lorsqu'il s'agit de définir une lettre dans l'Antiquité, en particulier chez Cicéron. C'est évidemment d'abord dans le cadre de la lettre amicale que cette formulation prend tout son sens, cette tension étant évidemment moins essentielle dans les courriers plus formels.

\section{La lettre comme cadeau : une compensation de l'absence?}

5 Chez le rhéteur Démétrios, qui fut peut-être, sur ses vieux jours, le maître de Cicéron ${ }^{15}$ et qui est surtout l'auteur d'un Sur le style ou Sur l'expression, la lettre amicale, présentée dans le cadre d'un exposé sur le style simple, n'est pas directement définie par la tension entre absence et présence. Démétrios circonscrit le discours épistolaire par opposition à d'autres genres de discours ${ }^{16}$ : par opposition au dialogue surtout 
(imitation d'une parole improvisée, ce que ne doit pas être la lettre), au théâtre (le dialogue théâtral étant marqué par les fréquentes disjonctions qui stimulent le jeu de l'acteur), au traité (l'en-tête «salut!» ne suffit pas à faire une lettre), à la plaidoirie (qui privilégie les périodes), à la dissertation de sciences naturelles (une lettre ne doit pas traiter de sujets trop techniques), aux discours pleins d'artifices faisant un large usage de maximes et d'exhortations (la lettre, elle, privilégie le proverbe). Le thème de l'absence et de la présence apparaît cependant en creux à travers l'idée, dont la fortune sera immense, que la lettre, portrait peint de l'âme du scripteur, est un cadeau, c'està-dire un substitut de la présence, une compensation de l'absence.

\section{La thématisation de la polarité entre absence et présence dans les lettres de Cicéron}

Chez Cicéron, on l'a déjà évoqué, la tension entre absence et présence est au cœur de la définition générique de la lettre amicale. L'écriture épistolaire est de fait, pour ainsi dire, victime d'un double problème d'absence: lors de la formulation, puisque le destinataire est absent ; lors de la réception, puisque, cette fois, l'épistolier est absent. Les formules cicéroniennes liées à cette double contrainte sont très souvent thématisées et ont été étudiées par Élisabeth Gavoille ${ }^{17}$ : topiques, elles disent le regret de l'absence («sache qu'en ce moment il ne me manque rien tant qu'un ami à qui je puisse confier mes soucis $\left.{ }^{18} »\right)$, elles soulignent l'effet de présence que produit la lecture d'une lettre ( je t'ai vu tout entier dans ta lettre ${ }^{19}$ », lui écrit Quintus après l'affranchissement de Tiron ${ }^{20}$ ), elles disent aussi l'illusion de présence que peut produire le fait d'écrire à l'ami (Video enim te et, quasi coram adsis [...] ${ }^{21}$ ). Liée à l'écriture de l'évidence ${ }^{22}$, la lettre peut aussi chercher à donner à voir l'épistolier dans des mises en scène de soi lisant ou écrivant (à la lueur de la lampe à huile, à l'aube, par exemple), se montrant à l'ami dans son activité de réflexion, dans le cadre de délibérations sur ce qu'il convient de faire ${ }^{23}$, entre monologue et dialogue (ego tecum tamquam mecum loquor ${ }^{24}$, lui arrive-t-il d'écrire à Atticus). Dans les lettres d'exil, on retrouve le lieu commun $\mathrm{du}$ correspondant considéré comme un autre soi-même (te quasi me alterum ${ }^{25}$ ) et celui de l'effet de présence, en particulier dans la correspondance avec Terentia, mais dans une missive où il recommande à sa femme de ne pas venir le voir et de rester à Rome pour défendre les intérêts familiaux. En Fam. 14, 2, 4, il prétend ainsi avoir son épouse devant les yeux nuit et jour. Le regret de l'absence et l'impression de voir le correspondant relèvent de lieux communs du discours amical. Ils se réfèrent à un idéal de l'amicitia qui hérite sur ce point de la philia grecque : celle-ci suppose, pour être source de bonheur et de vertu, selon Aristote ${ }^{26}$, une existence partagée, une communitas uitae pour le dire en termes cicéroniens.

\section{Absence et présence dans l'architecture formelle de la lettre}

7 L'effet de tension entre présence et absence apparaît aussi au niveau des mécanismes dialogiques de la lettre : l'inscriptio (Cicero Attico salutem dicit/dat) rappelle la distance entre des amis qui ne se nommeraient pas en coprésence, de même que la mention du lieu et de la date, mais aussi le recours au passé épistolaire ${ }^{27}$, qui crée des effets étonnants et spécifiques dans le repérage spatio-temporel. En effet, l'utilisation du passé dit «épistolaire » consiste à renvoyer aux coordonnées du récepteur. On peut citer l'exemple suivant, dans le corpus qui nous intéresse $(A t t .3,10,1)$ : acta quae essent 
usque ad a.d. VIII kalendas iunias cognoui ex tuis litteris; reliqua expectabam, ut tibi placebat, Thessalonicae. Quibus allatis facilius statuere potero ubi sim ${ }^{28}$. L'imparfait expectabam renvoie aux coordonnées du lecteur et non de l'épistolier : le transfert de cadre permet de donner à voir le scripteur depuis le point de vue du correspondant. Le passé suppose en effet que l'action est accomplie lorsque le lecteur aura la lettre entre les mains. En l'occurrence, le choix de l'imparfait, avec son aspect sécant, maintient la possibilité du contraire $^{29}$.

L'illusion de coprésence est parfois renforcée par le recours aux phatismes (mihi crede; ut scis), aux modulateurs de la force illocutoire (amabo te), aux apostrophes (on se souvient du triple mi frater dans la lettre Fam. 14, 2, 3 à Quintus ou du mea uita adressé à Terentia ${ }^{30}$ ), aux dialogues imaginés avec le correspondant, suivant un procédé évoquant la figure de la sermocinatio ${ }^{31}$, par exemple. Mais la comparaison a des limites évidentes. Du fait que la lettre ne dispose pas d'un canal oral, assorti d'« un matériel sémiotique composite", constitué de signes linguistiques et paralinguistiques (gestes, sons, etc.), l'émetteur, en l'absence d'une accessibilité perceptive mutuelle, ne peut manifester aussi facilement qu'il s'adresse à quelqu'un ni le signaler " par l'orientation de son corps, la direction dominante de son regard et la production de marqueurs verbaux d'allocution", en s'assurant, "par des coups d'œil intermittents, que l'autre écoute, et qu'il est bien branché sur le circuit communicatif " ${ }^{32}$. L'interlocution est souvent désirée dans la lettre, mais le copilotage, si important en face à face parce qu'il permet un remodelage de l'échange, ne peut être qu'imaginaire à l'écrit.

$9 \mathrm{Au}$ fond, toute lettre, comme l'écriture elle-même, aurait été inventée pour suppléer l'absence. À la croisée entre le monologal et le dialogal, la lettre familière en particulier, dont la fonction première est de maintenir le lien menacé par la distance, instaure une langue de la proximité, une Sprache der Nähe ${ }^{33}$, en exploitant à la fois ce qui permet de déplorer l'éloignement et l'absence tout en affirmant le pouvoir de coprésence que permet l'écriture.

\section{Les lettres d'exil ou le discours d'un absent}

Dans les lettres d'exil, ces différents marqueurs de la tension entre absence et présence sont employés, mais de manière spécifique, car l'absence, contrainte, est de nature différente, produisant un type original, non conventionnel, d'écriture épistolaire.

\section{Un cas de non-assistance à personne en danger}

11 L'absence, pour les lettres d'exil, avant d'être liée à la distance entre partenaires de l'échange, est due à une interdiction d'assistance imposée par une loi qui touche un seul des correspondants. L'un des aspects marquants de la seconde rogatio de Clodius est peut-être moins l'interdiction de s'approcher à moins de 500 milles de Rome que l'interdiction à tout citoyen de porter assistance à Cicéron par l'eau et le feu, interdiction qui contenait sans doute aussi un vieux fonds religieux (s'agissait-il d'une forme édulcorée de la sacratio ?). Voué, en quelque sorte, à une malédiction, l'individu ne pouvait compter ni sur les divinités protectrices des remparts ni sur les hommes. Une telle loi supposait par ailleurs une déchéance des droits civiques. Dans ses lettres, Cicéron précise la crainte que suscite l'interdiction par Clodius d'évoquer son cas même au sénat (Cic., Att. 3, 15, 6) : 
Ast tu scripsisti ad me quoddam caput legis Clodium in Curiae poste fixisse, ne referri neue dici liceret

Tu m'as écrit toi-même que Clodius avait affiché à la porte de la curie un certain article de sa loi portant défense de faire une proposition ou de prendre la parole sur mon rappel.

12 Les missives suggèrent aussi son amertume face à ceux qui refusent ou ont refusé ostensiblement de lui porter secours. Il y a le silence du lointain César ${ }^{34}$, qui part en cette même année pour la Gaule et avec lequel tentent de communiquer Varron ou Sestius. Il y a le silence de Pompée, d'abord enfermé dans sa villa d'Albe, qui a pu contribuer à l'affolement de Cicéron. Privé de l'aide des triumvirs, Cicéron est aussi exclu de tout dialogue direct avec eux : davantage que ses correspondants, Cicéron est du côté du non-dialogue, du soliloque ${ }^{35}$.

Les lettres à Atticus, à Terentia et à Quintus s'emploient à juger ceux qui ne l'ont pas aidé (Cic., Att. 3, 14, 1) :

Ex tuis litteris plenus sum expectatione de Pompeio, quidnam de nobis uelit aut ostendat.

Ta lettre m'a rempli d'impatience au sujet de Pompée: quels sont donc ses sentiments à mon égard, ou quels sont ceux dont il fait montre?

14 Le rôle joué par Atticus lui-même, ses conseils au début de l'affaire, font d'ailleurs eux aussi l'objet de vives critiques de la part de Cicéron; à plusieurs reprises, il ne manque pas de le lui signaler, comme cela apparaît à son retour de Rome (Cic., Att. 4,1,1) :

Cognoram enim, ut uere scribam, te in consiliis mihi dandis nec fortiorem nec prudentiorem quam me ipsum [...] nec etiam propter meam in te obseruantiam nimium in custodia salutis meae diligentem.

Car je n'ignorais pas, à dire vrai, que tu ne t'étais pas montré plus courageux que moi dans tes conseils, ni plus sage que moi, [...] et que tu n'avais pas témoigné beaucoup de zèle à te faire le gardien de mon salut.

Cicéron lui reproche aussi de ne pas l'aider avec les bons procédés : dans une lettre, il lui demande de le consoler et non de le blâmer ${ }^{36}$; dans une autre, il lui demande en revanche de cesser les consolations et de l'aider plutôt par ses conseils et son influence (gratia) à Rome ${ }^{37}$, à quoi il ajoute, dans les lettres suivantes, son besoin d'être informé dans le détail.

\section{Le thème du manque}

Cette recherche de relais susceptibles de lui porter assistance n'empêche pas Cicéron de manifester son desiderium : le manque causé par l'impossibilité d'être avec ses proches. L'exil se traduit ainsi également par l'expression d'un isolement amical et affectif : «Je suis toujours au même endroit, privé de toute conversation, sans aucune pensée ${ }^{38}$ ». Ailleurs, il rappelle à Atticus l'importance de ce manque causé par l'absence (Cic., Att. 3, $15,2)$ :

Ad primam tibi hoc scribo, me ita dolere ut non modo a mente non deserar sed id ipsum doleam, me tam firma mente ubi utar et quibuscum non habere. Nam si tu me uno non sine maerore cares, quid me censes qui te et omnibus?

À la première [lettre], je réponds ceci : oui, je souffre, mais sans que pour cela mes facultés intellectuelles m'abandonnent; bien plus, ce m'est précisément une souffrance que, faute d'occasion et de société, leur vigueur reste sans emploi. Ma seule absence n'est pas sans te causer du chagrin : quel doit être, dis-moi, le mien quand à ton absence s'ajoute celle de tous? 
17 L'absence et le manque (le desiderium) poussent alors l'épistolier à exprimer, de manière intensifiée, son lien affectif avec ses proches. C'est par exemple sensible dès les inscriptiones dans les lettres adressées à sa famille: Tullius s. d. Terentiae et Tulliae et Ciceroni suis. La coordination renforcée ainsi que le possessif suis signalent ici un attachement ostentatoire. On le voit aussi dans les séquences d'ouverture et de clôture, fortement marquées par la fonction phatique du langage, où Cicéron signale son émotion à la lecture de leur lettre («quand je vous écris ou quand je lis vos lettres, j'entre dans des crises de larmes à n'y plus pouvoir tenir $\left.{ }^{39} »\right)$; on le voit encore dans les apostrophes pathétiques ( $m i$ frater, mi frater, mi frater, dans la lettre Q.fr. 1, 3, 1 ou, à l'adresse de Terentia, avec l'expression poétique mea uita en Fam. 14, 4, 1). La distance et le manque d'information décuplent l'impression de séparation : le lien affectif est alors traduit par une inquiétude manifestée de manière expressive, voire excessive. Cicéron s'inquiète par exemple pour son frère qui rentre d'Asie après y avoir été propréteur. L'absence de nouvelles sur son voyage de retour lui fait craindre le pire : «mais la tristesse où me plongent mes malheurs, les craintes que j'éprouve pour mon frère me paralysent quand il s'agit d'écrire ${ }^{40} »$. Ces craintes le poussent à recommander les siens auprès de ceux en qui il a confiance, ne pouvant plus directement les protéger : " aide mon frère Quintus, qui peut être sauvé, protège Terentia et mes enfants ", écrit-il à Atticus ${ }^{41}$. Même type de recommandation à Quintus lui-même (Cic., Q. fr. 1, 3, 10) :

Filiam meam et tuam Ciceronemque nostrum quid ego, mi frater, tibi commendem? Quin illud maereo quot tibi non minorem dolorem illorum orbitas afferet quam mihi.

Qu'ai-je besoin, mon frère, de te recommander ma fille, qui est aussi la tienne, et notre cher Cicéron? Ce qui, plutôt, me tourmente, c'est de penser que tu ne souffriras pas moins que moi de les voir orphelins.

\section{Les amici, une présence de substitution à Rome}

18 L'absence impose à Cicéron une impossibilité d'agir. La correspondance ne cesse de souligner cette impuissance difficile à concilier avec le statut d'homme d'État qui avait été le sien. Si la lettre supplée l'absence, c'est ici au sens où elle délègue aux correspondants la faculté d'agir qui manque désormais à Cicéron. Les lettres à Atticus ou à Quintus s'achèvent inlassablement sur des actes de langage directifs ${ }^{42}$, portés par des verbes volitifs ${ }^{43}$ ou de requête, des impératifs ${ }^{44}$ qui marquent tantôt l'ordre, tantôt la requête. En témoignent, par exemple, les demandes répétées d'informations précises, qui viennent compléter les acta senatus qui lui parviennent. En voici quelques exemples : "pour moi, je te supplie de me donner sur toutes choses des détails et des précisions certaines » (ad me obsecro te ut omnia certa perscribas) ${ }^{45}$; " tiens-moi je te prie au courant de tout " (te oro ut ad me de omnibus rebus rescribas) ${ }^{46}$; "tâche donc de m'écrire tout en détail, après les avoir bien étudiées et scrutées » (quare fac ut omnia ad me perspecta et explorata perscribas) ${ }^{47}$.

19 L'information doit ainsi permettre de juger de la situation et d'alimenter son espoir (spes est un mot fréquent dans les lettres d'exil), mais aussi d'agir à bon escient. L' amicus a désormais le devoir d'incarner à Rome une présence de substitution. Son rôle est de compenser l'absence de Cicéron de deux manières: en renseignant son correspondant sur ce qui se passe dans l'Vrbs, en agissant à Rome pour œuvrer à son retour. C'est pourquoi Cicéron ne désire pas être rejoint dans l'immédiat par ses proches ou par Atticus. Contrairement aux situations classiques, la solution pour remédier à l'absence ne consiste pas à envisager de promptes retrouvailles (même si ce 
thème est esquissé dans les lettres qui nous occupent); il s'agit au contraire, pour les amici, d'intensifier leur présence à Rome. Cela explique que Cicéron, même s'il a du mal à l'avouer, demande à Terentia de ne pas le rejoindre. Il écrit davantage pour faire pression que pour manifester une réelle hésitation (Cic., Fam. 14, 4, 3) :

0 me perditum, o afflictum! Quid nunc rogem te ut uenias, mulierem aegram et corpore et animo confectam? Non rogem? Sine te igitur sim? Opinor, sic agam : si est spes nostri reditus, eam confirmes et rem adiuues; sin, ut ego metuo, transactum est, quoquo modo potes ad me fac uenias.

Ah quel désastre, quelle douleur! puis-je à présent te demander de me rejoindre, pauvre femme malade à bout de force et de courage? Ne pas te le demander? Rester privé de ta présence? Voici, je crois, ce que je ferai: si je puis espérer rentrer, je te prierai d'aider à la réalisation de cet espoir ; si, en revanche, comme je le crains, c'en est fait, arrange-toi par tous les moyens possibles pour venir me retrouver.

Il est clair que Cicéron préfère que Terentia reste à Rome et fasse son possible pour gérer le patrimoine et le préserver des pillages et des attaques. Cicéron le dit clairement à Atticus également : il est plus utile présent à Rome que présent auprès de lui : «je comprends que ta présence est utile là-bas (te istic prodesse), tandis qu'ici (hic) tu ne pourrais me consoler, même par tes propos ${ }^{48}$ ». Mais c'est avec Quintus que la situation est la plus tendue. Le jeune frère désirait rejoindre Cicéron après avoir été propréteur en Asie. Or Cicéron croit savoir qu'on prépare un procès de repetundis contre lui et lui demande donc de rentrer à Rome pour protéger les intérêts familiaux, refusant de le voir. Une brouille éclate. Cicéron doit se justifier auprès de lui et d'Atticus. Pour faire accepter son refus et son empressement de voir les siens le représenter et défendre ses intérêts à Rome, il use d'un argument qu'il juge imparable : il ne veut pas être vu dans cet état dégradé qui est le sien (Cic., Att. 3, 10,1):

uitaui ne [eum] uiderem, ne aut illius luctum squaloremque aspicerem aut me quem ille florentissimum reliquerat perditum illi afflictumque offerrem.

J'ai évité de le voir, ne voulant pas avoir sous les yeux l'affreux spectacle de sa douleur, ni me montrer à lui, moi qu'il avait laissé dans une situation si brillante.

\section{De l'invisibilité à l'absence à soi-même}

De fait, la spécificité de l'écriture des lettres d'exil est qu'il s'agit moins d'une conversation des absents que de l'écriture d'un absent. Cicéron, sans doute pour mieux convaincre ses proches et ses amis d'agir pour lui, ne cesse de donner à voir sa propre disparition, en recourant aux lieux communs de la miseratio ${ }^{49}$ (développés, par exemple, dans le De inuentione ${ }^{50}$ ) pour émouvoir ses correspondants et les pousser à agir ${ }^{51}$. Cicéron refuse ainsi la convention qui fait de l'écriture épistolaire une compensation de l'absence, soit qu'elle donne l'illusion d'être ensemble, soit qu'elle se présente comme une image de soi offerte à l'absent, soit encore qu'elle ouvre sur la perspective de retrouvailles prochaines. Il écarte les thèmes de la consolatio proposés par ses proches ${ }^{52}$ (ils pourraient avoir justement ce rôle de compensation) et, surtout, il refuse de donner à ses correspondants l'espoir d'être vu dans la condition de déchéance civique dans laquelle il se trouve. Le thème de l'absence est un moyen de faire pression sur les correspondants : l'absent des lettres d'exil est plus loin des siens qu'un mort ${ }^{53}$. En perdant la face, l'exclu de Rome, physiquement, juridiquement et symboliquement, n'a plus de rôle social, plus de persona, plus de visage. L'écriture épistolaire, loin d'avoir pour fonction de compenser l'absence, se donne alors pour rôle paradoxal de figurer cette absence, d'être la voix de celui qui n'a plus de face ${ }^{54}$. Il s'agit là d'une manière 
d'impressionner les correspondants et de rappeler leur devoir d'assistance, c'est-à-dire de présence, non auprès de lui, mais à Rome. C'est ainsi que l'on peut comprendre le lien entre l'exil et la mort fréquemment formulé par Cicéron. Le terme exsilium n'est jamais prononcé dans les lettres, Cicéron lui préférant les termes plus génériques et solennels de calamitas ou de pernicies ou encore de uulnus. Mais la mort est souvent évoquée à travers la tentation du suicide, ou par l'expression du regret de ne pas s'être tué au bon moment, ou encore à travers la mort comme métaphore de sa chute politique et sociale : «si dans mon malheur je laisse Quintus à l'abri du danger, il me semblera que je n'ai pas péri tout entier » (non me totum periisse abitrabor) ${ }^{55}$. Il assure par ailleurs à Quintus que celui-ci, s'il l'avait vu, ne l'aurait pas trouvé dans son état normal ; Cicéron est devenu plus « invisible » qu'un mort (Cic., Q. fr. 1, 3, 1) :

Ego te uidere noluerim? Immo uero me a te uideri nolui. Non enim uidisses fratrem tuum, non eum qui reliqueras, non eum quem noras, [...], ne uestigium quidem eius nec simulacrum sed quandam effigiem spirantis mortui.

La vérité, c'est que je n'ai pas voulu être vu de toi. Car ce n'est point ton frère que tu aurais vu, ce n'est point celui que tu avais laissé, que tu connaissais [...]. Non, de celui-là pas même la trace (uestigium), rien qui lui ressemblât (simulacrum), mais je ne sais quelle image d'un 'mort-vivant' ${ }^{56}$.

L'absence n'est plus le résultat d'un simple éloignement. C'est une disparition à soimême.

Le rapprochement entre absence et sentiment d'une identité perdue ou devenue problématique est opéré à plusieurs reprises en réponse aux reproches de manque de fermeté d'âme de la part d'Atticus. Ainsi, dans cette lettre: «je n'ai pas seulement perdu, en effet, mes biens (mea) et ceux que j'aime (meos), j'ai perdu ce qui était moimême (me ipsum). Oui, que suis-je à présent? (quid enim sum ?) ${ }^{57}$. Les propriétés foncières, qui désignent métonymiquement sa dignitas, les liens familiaux et le réseau des amici qui symbolisent sa gratia (la reconnaissance dont il bénéficie), c'est-à-dire ce qui constitue sa place dans les différents cercles de la communauté civique, sont associés à l'identité : perdidi [...] me ipsum. Dans la même lettre, un lien identique est suggéré entre l'identité (perdue) et la (dé)possession de ses biens, qui symbolisent sa dignitas (sa domus sur le Palatin représente sa dignité politique, tandis que ses uillae dans le Latium représentent sa dignité philosophique) (Cic., Att. 3, 15, 6) :

Quid de bonis? quid de domo? poteritne restitui? Aut si non poterit, egomet quo modo potero?

Et ma fortune, et ma maison? Pourra-t-on me les rendre? Si ce n'est pas possible, par quel moyen pourra-t-on me rendre [ce que] moi-même [j'étais]?

Cette dimension à la fois sociale et symbolique de la perte de soi est affirmée plus clairement encore dans ce passage (Cic., Att. 3, 10, 2):

Possum obliuisci qui fuerim, non sentire qui sim, quo caream honore, qua gloria, quibus liberis, quibus fortunis, quo fratre?

Puis-je oublier ce que j'ai été? Ne pas voir ce qu'à présent je suis? De quels honneurs je suis privé, de quelle gloire, de quels enfants, de quels biens, de quel frère?

Dans une telle société de l'honneur et de la honte, l'ancien consul a perdu la face, il ne veut être vu dans ces conditions et assimile sa condition à une mort.

L'absence est définie ici à partir de la perte de tous les espaces, du cercle familial à celui de l'existence publique, dans lesquels s'inscrit idéalement le citoyen et s'instaure son identité. Rome est symboliquement le lieu où se définissent les identités. À plusieurs reprises, l'épistolier évoque ses projets de voyage, en changeant d'itinéraire; il 
repousse son projet de s'installer en Épire, chez Atticus, envisage d'aller à Cyzique, sur les bords de la mer de Marmara, finalement reste plusieurs mois à Thessalonique, avant d'aller à Dyrrachium, n'écoutant manifestement pas les conseils d'Atticus qui le presse d'aller trouver refuge dans son domaine de Buthrote. S'il reste maître de ses choix de déplacement, il suggère également qu'aucun lieu n'est pleinement satisfaisant, que ses choix se font par défaut, ce qui accentue davantage encore cette stratégie d'invisibilité sur laquelle il insiste auprès de ses proches (Cic., Att. 3, 14, 2) :

Ego propter uiae celebritatem et cotidianam expectationem rerum nouarum non commoui
me adhuc Thessalonica. Sed iam extrudimur non a Plancio (nam is quidem retinet) uerum
ab ipso loco minime apposito ad tolerandam in tanto luctu calamitatem. In Epirum ideo, ut
scripseram, non ueni, quod subito mihi uniuersi nuntii uenerant et litterae, qua re nihil esset
necesse quam proxime Italiam esse.
Je n'ai pas bougé jusqu'ici de Thessalonique, parce que la route est très fréquentée,
et parce que j'attends chaque jour qu'il se produise du nouveau. Mais à présent j'en
suis chassé, non par Plancius (car lui, il me retient), mais par les inconvénients du
séjour même, qui est aussi peu fait que possible pour rendre le malheur tolérable à
un homme aussi affligé que moi. Si je ne suis pas allé en Épire comme je te l'avais
annoncé, c'est que soudain me sont venus des courriers et des lettres unanimes à
me dire qu'il n'y avait aucune nécessité de me trouver le plus près possible de
l'Italie.

Aucun lieu ne convient, soit qu'il soit désagréable, soit qu'il soit peu utile de s'y rendre, soit même qu'il soit dangereux d'y aller ou finalement impossible. L'absent n'est pas seulement celui qui est loin, mais celui dont la relation aux lieux a perdu de sa nécessité. Le choix des lieux de séjour est motivé a minima, souvent associé à la perspective d'aller plus loin (les bords de la mer de Marmara). À cette difficulté d'inscription dans l'espace peuvent être associées plusieurs mentions d'une certaine errance mentale ou, en tout cas, d'une difficulté à faire des choix : "L'incohérence de mes lettres te montre assez, je pense, le trouble de mon esprit (motum mentis meae) ${ }^{58}$ ». Tout se passe comme si l'épistolier se montrait incapable d'endosser les quatre rôles (personae) dont il sera question, quelques années plus tard dans le De officiis, son dernier ouvrage philosophique. Dans le cadre d'un développement sur l'honestum, Cicéron, reprenant une théorie développée par Panétius de Rhodes, représentant du stoïcisme moyen au siècle précédent, postule que tout individu doit revêtir quatre personae (« masques », « rôles »), qui sont comme quatre aspects de sa subjectivité ${ }^{9}$ : il faut être conforme à la raison universelle (première persona), à son tempérament (deuxième persona), savoir s'adapter aux circonstances (troisième persona), enfin savoir se déterminer quand plusieurs choix sont possibles (c'est la quatrième persona). Or les lettres d'exil ${ }^{60}$ montrent un épistolier qui refuse qu'on lui fasse entendre raison (il demande à Atticus de n'être ni blâmé ni consolé davantage), un épistolier qui n'accepte pas les circonstances en refusant d'accepter l'idée qu'il puisse y avoir des cas semblables ou comparables à sa propre histoire ( j'ai été victime d'une catastrophe incroyable, unique ", incredibili et singulari calamitate), qui ne sait pas que décider ("je reste à Thessalonique, mortellement inquiet et n'osant rien faire $\left.{ }^{61} »\right)$, ou regrette ses choix passés ${ }^{62}$, déléguant partiellement ${ }^{63}$ cette faculté à ses amici, comme l'indique l'ensemble des passages où Cicéron invite ses correspondants à évaluer par eux-mêmes la situation ${ }^{64}$ et à prendre des initiatives ("à toi de voir tout ce qui se passe et de diriger la manœuvre ${ }^{65} »$ ). En un sens, l'absence est aussi une absence à soi-même, dans la mesure où Cicéron, refusant les consolations qu'on lui adresse, ne semble pas prêt à 
une quelconque cura sui. Incapable de retrouver l'égalité à soi-même, c'est aux amici de l'aider à retrouver un visage (Cic., Att. 3, 15, 8) :

meque, ut facis, uelis esse aliquem, quoniam qui fui et qui esse potui iam esse non possum. et aie la volonté, comme tu le fais, de faire que je sois quelqu'un, puisque ce que je fus, et ce que j'aurais pu être, je ne puis plus l'être.

\section{La requalification de l'absence après l'exil}

\section{Retour à Rome, retour à la visibilité}

La première lettre écrite de Rome après son exil est pour nous adressée à Atticus, qui est certainement resté en Épire. Elle permet de confirmer comment s'articule le rapport entre identité et présence au sein de l'espace civique. Le retour à Rome est d'abord présenté comme une nouvelle naissance, une image qui s'oppose à celle de l'exil comme mort que nous avions rencontrée : «quelque chose comme une nouvelle vie commence pour moi » (alterius uitae quoddam initium ordimur) ${ }^{66}$. Cette nouvelle vie résulte de la reconquête des différents éléments qui permettent son inscription dans la cité (Cic., Att. 4, 1, 3) :

Nos adhuc, in nostro statu quod difficillime recuperari posse arbitrati sumus, splendorem nostrum illum forensem et in senatu auctoritatem et apud uiros bonos gratiam magis quam optamus consecuti sumus.

Jusqu'à présent, chose que je croyais la plus difficile à récupérer dans ma situation, j'ai reconquis ma place brillante au forum, mon autorité au sénat et de la reconnaissance auprès des gens de bien, au-delà de ce que nous pouvons souhaiter.

Il lui reste cependant à récupérer son patrimoine (res familiaris) abîmé et amoindri par les pillages. L'ancrage dans la cité n'est pas ici un rapport au sol mais un rapport à la visibilité et à la reconnaissance. Cicéron insiste dans sa lettre sur le voyage qui le ramène de Brindes à Rome. Il souligne le merveilleux rassemblement unitaire autour de lui (incredibili concursu Italiae ${ }^{67}$ ), l'empressement des différents ordres à le rencontrer («il n'y eut pas un homme connu de mon nomenclator, à quelque ordre qu'il appartînt, qui ne vînt à ma rencontre $\left.{ }^{68} »\right)$; arrivé à Rome, les temples sont couverts de la foule des petites gens : le peuple dans toutes ses composantes applaudit. L'exil était une mort, le retour est une renaissance; l'exil était un parcours de lieux non motivés, le retour le conduit comme un triomphateur jusqu'au Capitole; l'exil imposait une perte de cohérence et d'unité, le retour est marqué par le sceau de l'unanimité; l'exil le contraignait à une invisibilité, le retour est un spectacle. La restitution de sa dignitas rend à Cicéron une unité et une raison sociale, une persona, un masque cohérent, défini par le regard que l'on peut enfin poser sur lui. L'ancrage dans la cité est moins ici un attachement au territoire que la possibilité d'être reconnu. L'unité du peuple dans sa reconnaissance témoigne de l'unité retrouvée pour le personnage. Après son retour, l'affirmation de sa présence au sénat et face au peuple ne se dément pas : il y a bien sûr les discours Post reditum, les 5 et 7 septembre, mais aussi une séance au sénat où il est question de la cherté de la vie ; c'est Cicéron, par ailleurs mis en cause par Clodius, qui propose un décret et qui ensuite, après la lecture du sénatus-consulte où son nom est applaudi, fait un discours devant le peuple ${ }^{69}$. 


\title{
Rome absente à elle-même
} et sur son absence de Rome. L'un des points de son argumentation est le suivant: non, Cicéron n'a pas connu l'exil, ce n'est pas lui qui était absent de Rome, c'est Rome qui était absente à elle-même (Cic., Red. Sen. 34) :

Sed cum uiderem me non diutius quam ipsam rem publicam ex hac urbe afuturum, neque ego illa exterminata mihi remanendum putaui, et illa, simul atque reuocata est, me secum pariter reportauit. Mecum leges, mecum quaestiones, mecum iura magistratuum, mecum senatus auctoritas, mecum libertas, mecum etiam frugum ubertas [...] afuerunt.

Mais comme je voyais que je ne serais pas absent de cette ville plus longtemps que la République elle-même, je n'ai pas cru devoir rester une fois celle-ci détruite; et j'ai pareillement été rappelé auprès d'elle dès qu'elle a été rétablie. Les lois, la justice, les droits des magistrats, l'autorité du sénat, la liberté des citoyens, même la fertilité des campagnes [...], tout cela a été banni avec moi.

L'arrivée de Clodius au pouvoir traduisait un exil de la République tout entière. Le retour de Cicéron signalait au contraire le retour de la République à elle-même. Les discours de remerciement au sénat et au peuple permettent de donner une visibilité historique à ses tempora. L'absence change de sens : elle n'est pas le fait d'un individu mais d'un État dans son ensemble.

\section{Visages en pleurs : dignité philosophique et impact politique des larmes}

Les correspondants de Cicéron l'engagent à plusieurs reprises à faire davantage preuve de fermeté durant la période de l'exil. Celui-ci répond le plus souvent que sa détresse est justifiée par le caractère inédit de la situation. Mais c'est surtout dans la correspondance avec Terentia et avec Quintus que le thème des larmes est développé par l'épistolier. Les pleurs signalent-ils un défaut de présence à soi-même, sont-ils le signe d'une errance mentale, comme voudraient le faire croire ses détracteurs à Rome ? Dans le De domo sua, Cicéron n'hésite pas à rappeler qu'il est juste de pleurer sur un drame aussi effroyable et que le sage insensible à la douleur n'est pas un modèle (Cic., Dom. 98) ${ }^{70}$ :

\begin{abstract}
disturbari tecta, diripi fortunas, patriae denique causa patriam ipsam amittere [...] haec omnia subire conseruandorum ciuium causa, atque id cum dolenter adsis non tam sapiens quam ii qui nihil curant, sed tam amans tuorum ac tui quam communis humanitas postulat, ea laus praeclara atque diuina est.

voir sa maison démolie, ses biens pillés, enfin perdre sa patrie pour la défendre [...], subir tous ces maux pour le salut de ses concitoyens, et cela en souffrant sans être aussi sage que celui qui n'est touché par rien, mais avec autant d'amour pour les siens et pour soi-même qu'il est naturel à l'homme de ressentir : voilà qui est digne d'un éloge capable de conférer gloire et immortalité divine.
\end{abstract}

31 Dans son discours de remerciements au sénat, il souligne que les larmes sont aussi une force politique, qu'il n'avait que sa famille et les larmes de celle-ci pour défendre sa cause, quand d'autres grands exilés bénéficiaient de soutiens considérables (il cite en particulier le cas de Marius) (Cic., Red. Sen. 37) :

sed unus frater, qui [...] lacrimis et cotidianis precibus desiderium mei nominis renouari et rerum gestarum memoriam usurpari coegit. 
mon frère est le seul qui [...] par ses larmes, par les supplications continuelles, a fait en sorte que mon nom soit l'objet d'un manque et que le souvenir de mes services rendus soit évoqué.

Les proches de Cicéron apparaissent dans ses discours postérieurs à l'exil comme les relais de ses propres larmes, offrant sur la scène publique un visage de substitution. Les larmes légitimes sur le plan philosophique s'avèrent aussi d'une redoutable efficacité dans le cadre d'une politique de la présence, telle que Cicéron et les siens la construisirent pendant ces seize mois de relégation.

\section{Conclusion}

Les lettres d'exil privilégiaient une forme paradoxale de figuration de soi. Dans la perspective d'une rhétorique de la miseratio, Cicéron insistait sur son absence comme une forme de dissipation de soi, mettant en évidence sa propre disparition, son invisibilité et sa perte d'unité. Il se décrivait, somme toute, comme incapable d'endosser les quatre personae dont il formula la théorie, après Panétius, dans le $D e$ officiis, quelques années plus tard. Bien plus qu'une conversation des absents, les lettres d'exil se désignent elles-mêmes comme le soliloque d'un absent, exclu du dialogue romain, comme la parole de celui qui, ayant perdu la face, n'a plus de visage. À son retour, il est temps pour lui de figurer l'exil de manière positive, de l'intégrer à la fois dans son histoire et dans celle de Rome, et de faire de son retour un retour de Rome à elle-même. Les projets historiographiques qu'il conçoit autour de son consulat et de son exil participent de cette visibilité qu'il accepte à présent de donner à ses tempora.

\section{BIBLIOGRAPHIE}

\section{Textes anciens}

Aristote, Éthique à Nicomaque, préf. et commentaires de R. Arnaldez, texte trad. par J. Defradas, éd. et annoté par M. Defradas et Fr. Defradas-Colmez, Agora 98, Paris, 1992.

Cicero, Epistulae ad Quintum fratrem et M. Brutum, ed. by D. R. Shackleton Bailey, Cambridge, 1980.

Démétrios, Sur le style, texte établi et trad. par P. Chiron, CUF 353, Paris, 1993.

Lettres pour toutes circonstances. Les traités épistolaires du Pseudo-Libanios et du Pseudo-Démétrios de Phalère, introd., trad. et commentaire par P.-L. Malosse, La Roue à livres 42, Paris, 2004.

\section{Textes modernes}

BALLY Ch. 1944, Linguistique générale et linguistique française, $2^{\mathrm{e}}$ éd., Paris [1 ${ }^{\text {re }}$ éd. : 1932].

BERNARD J.-E. 2013, La sociabilité épistolaire, Babeliana 16, Paris. 
ChARAUDEAu P., MAINGUENEAu D. (dir.) 2002, Dictionnaire d'analyse du discours, Paris.

ClAASSEN J. M. 1992, « Cicero's Banishment: Tempora et Mores », AC 35, p. 19-47.

- 1996a, « Dio's Cicero and the Consolatory Tradition », Papers of the Leeds International Latin Seminar, IX, Arca 34, Leeds, p. 29-45.

- 1996b, « Exile, Death and Immortality: Voices from the Grave », Latomus 55, p. 571-590.

- 1999, Displaced Persons. The Literature of Exile from Cicero to Boethius, Londres.

CORDIER P. 1995, « La lettre et l'amicitia », in Fl. Dupont (éd.), Paroles romaines, Travaux et mémoires. Études anciennes 12, Nancy, p. 25-34.

DE GIORGIO J.-P. 2008, « 'Je t'ai vu tout entier dans tes lettres'. Humanitas, portrait d'âme et persuasion dans l'art de la conversation tardo-républicaine », in P. Laurence,

Fr. Guillaumont (éds), Epistulae antiquae. V, Actes du $V^{e}$ colloque international « L'épistolaire antique et ses prolongements européens ", Université François-Rabelais, Tours, 6-7-8 septembre 2006, Louvain Paris, p. 101-114.

DE GIORGIO J.-P., NDIAYE E. 2012, " Figurer l'exil : Cicéron entre Histoire et histoire personnelle », in Fr. Guillaumont, P. Laurence (éds), La présence de l'histoire dans l'épistolaire, Tours, p. 385-402.

DESSALLE S. 1986, «Introduction : histoire du mot énonciation », Histoire, épistémologie, langage 8/2, p. 3-22.

FOUCAUlT M. 2001 [1983], « L'écriture de soi », in Dits et écrits, 1954-1988, II, Quarto, Paris, texte n 329 , p. $1234-1249$.

GARCEA A. 2002, "L'interaction épistolaire entre dialogue in absentia et in praesentia chez Cicéron », in A. M. Bolkeistein, C. H. M. Kroon, H. Pinkster, H. W. Remmekink, R. Risselada (éds), Theory and Description in Latin linguistics. Selected Papers from the XIth International Colloquium on Latin Linguistics, Amsterdam, June 24-29, 2001, Amsterdam Studies in Classical Philology 10, Amsterdam, p. 123-138.

- (éd.) 2003, Colloquia absentium. Studi sulla communicazione epistolare in Cicerone, Linguistica, Turin.

- 2004, «Le langage des émotions dans les lettres d'exil de Cicéron », in L. Nadjo, É. Gavoille (éds), Epistulae antiquae. III, Actes du III $I^{e}$ colloque international « L'épistolaire antique et ses prolongements européens », Université François-Rabelais, Tours, 25-27 septembre 2002, Louvain - Paris, p. 153-167.

- 2005, Cicerone in esilio. L'epistolario e le passioni, Spudasmata 103, Hildesheim - New York.

- 2006, « Cicéron hors de Rome. Les passions et l'identité de l'exilé », in P. Galand-Hallyn,

C. Lévy (éds), Vivre pour soi, vivre pour la cité, de l'Antiquité à la Renaissance, Rome et ses renaissances, Paris, p. 223-232.

GAVoILLE É. 2000, «La relation à l'absent dans les lettres de Cicéron à Atticus », in L. Nadjo, É. Gavoille (éds), Epistulae antiquae. Actes du I ${ }^{e r}$ colloque « Le genre épistolaire antique et ses prolongements ", Université François-Rabelais, Tours, 18-19 septembre 1998, Louvain - Paris, p. 153-176. GROS F. 2006, « Le souci de soi chez M. Foucault », in P. Galand-Hallyn, C. Lévy (éds), Vivre pour soi, vivre pour la cité, de l'Antiquité à la Renaissance, Rome et ses renaissances, Paris, p. 19-30.

KELLY G. P. 2006, A History of Exile in the Roman Republic, Cambridge.

KERBRAT-ORECCHIONI C. 1995, Les interactions verbales, I, $2^{\mathrm{e}}$ éd. mise à jour, Linguistique, Paris [1 ${ }^{\text {re }}$ éd. : 1990]. 
KOCH P., OESTERREICHER W. 1985, « Sprache der Nähe - Sprache der Distanz. Mündlichkeit und Schriftlichkeit im Spannungsfeld von Sprachtheorie und Sprachgeschichte », Romanistisches Jahrbuch 36, p. 15-43.

LÉOVANT-CIRÉFICE V. 2014, « Les lettres de l'exil dans la correspondance de Cicéron : une thérapie de la douleur? », VL 189-190, p. 54-69.

LÉVY C. 2006, «Y a-t-il quelqu'un derrière le masque ? À propos de la théorie des quatre personae chez Cicéron ", in P. Galand-Hallyn, C. Lévy (éds), Vivre pour soi, vivre pour la cité, de l'Antiquité à la Renaissance, Rome et ses renaissances, Paris, p. 45-58.

LÉVY C., PERNOT L. (éds) 1997, Dire l'évidence. Philosophie et rhétorique antiques. Actes du colloque de Créteil et de Paris (24-25 mars 1995), Paris - Montréal.

MELLET S. 1988, L'imparfait de l'indicatif en latin classique. Temps, aspect, modalité. Étude synchronique dans une perspective énonciative, BIG, Louvain - Paris.

MOREAU P. 1987, « La lex Clodia sur le bannissement de Cicéron », Athenaeum 65/3-4, p. 465-492.

NARDUCCI E. 1997, «Perceptions of Exile in Cicero: The Philosophical Interpretation of a Real Experience », AJPh 118/1, p. 55-73.

NICHOLSON J. 1992, Cicero's Return from Exile. The Orations Post Reditum, Lang Classical Studies 4, New York - Berne.

RAUZY E. 2003, «Les représentations mentales mises sur pied dans la lettre par Cicéron », in A. Garcea (éd.), Colloquia absentium. Studi sulla communicazione epistolare in Cicerone, Linguistica, Turin, p. 101-121.

VIOLI P. 1988, «Présence et absence. Stratégies d'énonciation de la lettre », in La lettre. Approches sémiotiques, Interdisciplinaire 9, Fribourg, p. 27-35.

\section{NOTES}

1. Violi 1988.

2. Ibid., p. 27.

3. Rien à voir, donc, avec la dimension philosophique d'une telle tension, telle qu'elle a pu être développée dans la longue tradition de la métaphysique de la présence (avec Husserl et Heidegger), voire dans la réflexion derridienne sur la « différance ».

4. Voir par ex. Bally 1944.

5. Pour une histoire du terme "énonciation ", lié à des notions de rhétorique, mais qui relève en France de la grammaire et de la logique, avant d'être annexé au domaine de la linguistique, voir Dessalle 1986. Cette esquisse d'une histoire du terme permet de voir ce que Benvéniste doit à Weil et à Bally.

6. Voir l'exemple de lettre amicale que donne Ps.-Démétrios dans son manuel répertoriant les grandes catégories de lettres, intitulé Typoi epistolikoi (fin $\mathrm{III}^{\mathrm{e}} \mathrm{s}$. - début IV $\mathrm{I}^{\mathrm{e}}$ s. apr. J.-C.). L'absence y apparaît comme le motif le plus évident pour débuter une telle lettre : «Bien que je me trouve séparé de toi par une grande distance, je ne le suis que physiquement, car non, il ne sera jamais possible que je t'oublie, ni que j'oublie que, dès l'enfance, nous avons été élevés ensemble en bonne entente [...]». Nous citons ici la traduction de P.-L. Malosse, 2004, p. 56.

7. Sur cette discipline qui englobe, en France, divers champs disciplinaires (linguistique liée à l'analyse de la conversation, sociologie, philosophie), voir Charaudeau, Maingueneau (dir.) 2002, p. 41 sq. 
8. On peut signaler notamment l'ouvrage collectif de Garcea (éd.) 2003. Voir également l'article de synthèse que le même auteur consacre à cette question : Garcea 2002.

9. Cic., Fam. 2, 4, 1 : Epistolarum genera multa esse non ignoras, sed unum illud certissimum, cuius causa inuenta res ipsa est, ut certiores faceremus absentis, si quid esset quod eos scire aut nostra aut ipsorum interesset. Huius generis a me profecto non exspectas [...]. Reliqua sunt epistularum genera duo, quae me magnopere delectant, unum familiare et iocosum, alterum seuerum et graue. Nous reprenons le plus souvent, pour la correspondance, la traduction proposée par la CUF, généralement avec quelques modifications.

10. Cordier 1995. Sur le litterarum officium, voir également la synthèse de Bernard 2013, p. 71-104. L'auteur note en particulier, p. 73 : «La correspondance s'insère parmi les obligations de la vie sociale à Rome, il s'agit d'un officium de la vie privée comme de la vie publique qui contribue à maintenir les liens de solidarité et d'amitié au sein de la classe dirigeante romaine, dans une société fondée sur les réseaux clientélaires, à l'échelle d'un Empire, où les amis et les proches sont précisément désignés sous le nom de necessarii pour marquer le caractère réciproque et obligatoire de leurs relations, y compris et surtout dans le cas d'une grande intimité entre les individus ». L'officium consistant à informer est le mieux représenté dans la correspondance, mais il peut aussi s'agir de consoler, de recommander, de conseiller ou, tout simplement, d'entretenir la familiaritas.

11. Cic., Q. fr. 1, 4,4 .

12. Sur les deux rogationes de Clodius concernant cet épisode de l'exil, voir Moreau 1987 ; Kelly 2006 et, en dernier lieu, l'analyse de Michèle Ducos dans ce volume.

13. Pour un éclaircissement de cette notion, voir Gros 2006, p. 22-23.

14. Par ex., dans la correspondance du printemps 59, Cicéron, marginalisé par la formation du premier triumvirat et l'élection de César au consulat, affirme à Atticus (o noster Tite) avoir désormais suffisamment satisfait au philosophe péripatéticien Dicéarque (qui semblait avoir prôné la suprématie de la vie active sur tout autre mode d'existence) et jure vouloir se limiter désormais à la vie contemplative.

15. Chiron (éd.) 1993, p. xxxix.

16. Demetr., Eloc. 223-233.

17. Gavoille 2000.

18. Cic., Att. 1, 18, 1 : Nihil mihi nunc scito tam deesse quam hominem eum quocum omnia quae me cura aliqua afficiunt una communicem [...].

19. Cic., Fam. 16, 16, 2 : Te totum in litteris uidi.

20. De Giorgio 2008.

21. Cic., Att. 5, 18, 3 : «Je te vois et, comme si tu étais en face de moi [...]».

22. Lévy, Pernot (éds) 1997.

23. Les structures syntaxiques à dilemme dans les lettres d'exil font l'objet d'une étude spécifique par Ch. Guérin dans les actes de cette journée. Ce type de structure représente les scénarios possibles d'une situation mais signalent aussi la position de l'exilé, privé de toute possibilité d'action. Comme le rappelle Garcea 2004, p. 160 : "Cicéron se montre ainsi sous le poids de l'adversité, comme s'il se trouvait toujours à un carrefour et qu'il devait modifier ses choix et ses attentes en fonction de circonstances qu'il ne contrôle pas. ».

24. Cic., Att. 8, 14, 2.

25. Cic., Att. 3, 15, 4.

26. Arist., EN 1171a (trad. J. Defradas, 1992, p. 236-237): «La présence des amis est donc évidemment souhaitable en toutes circonstances. N'est-il pas vrai que, de même que les amants aiment tout particulièrement voir leur bien-aimé et préfèrent à toutes les autres cette sensation, comme si l'existence et le devenir du désir en procédaient, de même les amis souhaitent tout particulièrement vivre ensemble. »

27. Voir Mellet 1988 et Rauzy 2003. 
28. Cic., Att. 3, 10, 1.

29. Mellet 1988, p. 200.

30. Cic., Fam. 14, $2,3$.

31. Sur cette figure, voir Rhet. ad Her. 4, 65. L'usage de ces dialogues fictifs apparaît par ex. dans la lettre Q. fr. 1, 4, 4.

32. Kerbrat-Orecchioni 1995 [1990], p. 18.

33. Koch, Oesterreicher 1985, p. 15-43.

34. Sur le rôle de César et des différents membres de la classe politique dans cet épisode de l'exil, voir Nicholson 1992.

35. Voir sur ce point Garcea 2004, p. 155, et Claassen 1999, p. 27 (cité par A. Garcea) : "Although he frequently aknowledges receipt of letters, the impression is of a drawn-out monologue, not conversation. Cicero is the 'exiled I', the center of a dislocated universe where the hub is out of kilter ».

36. Cic., Att. 3, 10, 3: haec eo scripsi ut potius releuares me, quod facis, quam ut castigatione aut obiurgatione dignum putares.

37. Cic., Att. 3, 11, 2 : Tu me, ut facis, opera, consilio, gratia iuua ; consolari iam desine.

38. Cic., Att. 3, 12, 3 : Ego etiam nunc eodem in loco iaceo sine sermone ullo, sine cogitatione.

39. Cic., Fam. 14, 4, 1 : cum aut scribo aut uestras lego, conficior lacrimis sic ut ferre non possim.

40. Cic., Att. 3, 8, 4 : Me et meorum malorum maeror et metus de fratre in scribendo impedit.

41. Cic., Att. 3, 19, $3:$ Q. fratrem qui potest esse saluus sustentes, Terentiam liberosque meos tueare [...].

42. Cet aspect a été étudié en détail par Garcea 2004, p. 162 sq.

43. Par ex. : cupio, uelim (avec subjonctif d'atténuation : Att. 3, 4; 3, 10, 3; 3, 12, 2; 3, 13, 3 ; 3, 14, $1 ; 3,15,8 ; 3,17,3 ; 3,20,3 ; 3,22,4 ; 3,23,4$ et $5 ; 3,24)$.

44. Par ex. : hoc scito (Fam. 14, 4, 3), fac (Att. 3, $4 ; 3,13,3 ; 3,15,7 ; 3,18,2$ ).

45. Cic., Att. 3, 11, 2.

46. Cic., $Q$. fr. 1, 4,5 .

47. Cic., Att. 3, 15, 8

48. Cic., Att. 3, 12, 3.

49. Garcea 2006, p. 225-226, montre comment les lettres d'exil s'appuient d'une part sur la description d'événements susceptibles de susciter la pitié (responsabilité de la fortune, séparation des êtres proches, etc.) et d'autre part sur la mise en valeur des mérites du miserandus. 50. Cic., Inv. 1, 106-109.

51. Voir sur ce point les analyses de Garcea 2005.

52. Il repousse par ex. l'argument topique des consolationes insistant sur le caractère inéluctable du fatum (Fam. 14, 1, 1, à Terentia : quae si, ut tu scribis, fato facta putarem, ferrem paulo facilius) ; il précise à Quintus que, désormais, ni la prudentia ni aucune doctrina philosophique ne lui sont d'aucun secours (Q.fr. 1, 3, 5). Claassen 1992 parle de certaines réponses de Cicéron à ses destinataires comme autant d'anti consolationes. Il est significatif que Dion Cassius (38, 18-19), en revenant sur l'exil de Cicéron, ait imaginé une conversation philosophique entre Philiscus et lui en Macédoine, le premier développement des thèmes philosophiques courants de la consolation. Voir Claassen 1996a. Cicéron semble ainsi refuser toute forme de cura sui, pour reprendre une notion analysée par Foucault 2001 [1983]. Le dossier a été récemment repris par Léovant-Ciréfice 2014.

53. Cette idée est développée par Claassen 1996b. Cicéron la développe particulièrement dans la lettre à son frère $Q$. fr. 1, 3, 6 : si Cicéron avait choisi de mourir au bon moment, sa mort aurait été plus utile à son frère que cette survie qui l'empêche de prendre la défense des siens. S'il conçoit désormais des retrouvailles avec Terentia, c'est pour connaître la mort dans ses bras (Fam. 14, 4, 1 : ego uero te quam primum, mea uita, cupio uidere et in tuo sinu emori).

54. On peut ici renvoyer à l'usage que les études de pragmatique et les analyses de l'interaction font de ce terme, entendu au sens de "prestige», "honneur» ou "dignité». Dans une 
interaction, chacun cherche à conserver, voire à accroître son territoire et sa face (face-want, désir et besoin de face). Mais ce désir est souvent contrarié dans les interactions et nécessite une série de négociations. Voir la mise au point de C.Kerbrat-Orecchioni dans Charaudeau, Maingueneau (dir.) 2002, p. 259-261. Dans une certaine mesure, cette notion peut être ici mise en relation avec celle de persona. Voir De Giorgio, Ndiaye 2012.

55. Cic., Att. 3, 13, 2.

56. Cette expression renvoie peut-être à l'univers de la tragédie. L'image du mort-vivant apparaît par ex. chez Sophocle, Ant. 1067 et Ph. 1081, comme le note D. R. Shackleton Bailey, 1980, p. 166.

57. Cic., Att. 3, 15, 2 : Desidero enim non mea solum neque meos sed me ipsum. Quid enim sum?

58. Cic., Att. 3, 8, 4 : Ex epistularum mearum inconstantia puto te mentis meae motum uidere.

59. Cic., Off. 1, 107-118. Voir sur ce passage Lévy 2006.

60. Nous nous inspirons ici des remarques de Garcea 2006, p. 230-232.

61. Cic., Att. 3, 8, 2 : maneo Thessalonicae suspensus nec audeo quicquam.

62. Le regret le plus important concerne la décision de prendre la fuite après la première rogatio de Clodius, sur le conseil des amici. Voir par ex. Att. 3, 15, 4.

63. L'épistolier ne se prive pas, en effet, de critiquer les initiatives des amici : dans la lettre Att. 3, 23 , il s'en prend au projet de loi déposé par les tribuns de la plèbe pour obtenir son rappel; dans la lettre Att. 3, 24, il reproche à Atticus d'avoir consenti à l'organisation des commandements provinciaux des futurs consuls de 57.

64. Par ex. Q. fr. 1, 4, $5:$ Tu et quid opus sit et quid sit uidebis omnino.

65. Cic., Att. 3, 8, 4 : tu ista omnia uide et guberna.

66. Cic., Att. 4, $1,8$.

67. Cic., Att. 4, 1,4 .

68. Cic., Att. 4, 1, 5.

69. Cic., Att. 4, 1, 6 .

70. Narducci 1997.

\section{RÉSUMÉS}

Toute lettre amicale peut se définir à Rome comme la conversation des absents. Mais la tension entre absence et présence prend un tour particulier dans le cas des lettres d'exil de Cicéron. Elle pose ici le problème de la «figuration de soi » dans une situation d'éloignement forcé, humiliante pour l'homme d'État et le citoyen privé de droits, et, plus largement, celui de la difficile définition d'une identité hors de la cité.

A letter ad familiares in ancient Rome was defined as a conversation between separated people. But the nature of presence and absence is different in the case of the letters written in the period of exile. The stateman, deprived of his civic rights, has to face the problem of the Self outside Rome. 
INDEX

nomsmotscles César, Cicéron, Démétrios de Phalère, Dion Cassius, Panaitios de Rhodes, Rhétorique à Herennius, Sophocle, Varron

Keywords : Exile, correspondences, identity, Rome, absence, presence

Mots-clés : Exil, épistolaire, identité, Rome, absence, présence

\section{AUTEUR}

JEAN-PIERRE DE GIORGIO

Université Clermont-Ferrand II, CELIS (EA 1002) 